\title{
Molecular mechanisms of melatonin in the reversal of LPS-induced EMT in peritoneal mesothelial cells
}

\author{
SHUAISHUAI SHI ${ }^{1,2}$, YIQIANG ZHANG ${ }^{3}$, WENBIN WEN $^{2}$, YINE ZHAO $^{2}$ and LIN SUN $^{1}$ \\ ${ }^{1}$ Department of Nephrology, The Second Xiangya Hospital, Kidney Institute of Central South University, Changsha, \\ Hunan 410011; ${ }^{2}$ Department of Nephrology Changzhi Medical College Affiliated Heji Hospital, Changzhi, Shanxi 046011; \\ ${ }^{3}$ Department of Biochemistry Changzhi Medical College Changzhi, Changzhi, Shanxi 046000, P.R. China
}

Received August 10, 2015; Accepted July 28, 2016

DOI: $10.3892 / \mathrm{mmr} .2016 .5744$

\begin{abstract}
Peritoneal dialysis (PD)-associated peritoneal fibrosis is a serious complication in patients with chronic renal failure on dialysis maintenance. Studies have shown that patients on long-term PD have chronic inflammation. The epithelial-to-mesenchymal transition (EMT) induced by inflammation is a major cause of peritoneal fibrosis and dysfunction. As a potent antioxidant property, melatonin has an antifibrotic effect. The present study investigated the effects of melatonin on lipopolysaccharide (LPS)-induced EMT and examined the molecular mechanisms in peritoneal mesothelial cells using western blotting, reverse transcription-polymerase chain reaction and immunofluorescence staining. The results of the study found that melatonin inhibited LPS-induced morphological changes, decreased the expression of LPS-induced markers of EMT, including vimentin and $\alpha$-smooth muscle actin, and increased the expression of E-cadherin. In addition, it was found that the action of melatonin was mediated through the inactivation of the Toll-like receptor (TLR)4/c-Jun N-terminal kinase and TLR4/nuclear factor- $\mathrm{BB}-$ Snail signaling pathways. Thus, these data provided novel insight into the mechanisms underlying the function of melatonin in peritoneal mesothelial cells during the processes of EMT, and may provide a theoretical basis for the treatment of peritoneal fibrosis.
\end{abstract}

\section{Introduction}

As one of the major life-threatening diseases, end-stage renal disease (ESRD) is increasing in developed and the developing countries, and imposes a major social and economic burden on the majority of countries $(1,2)$. Patients with ESRD have three principal choices for renal replacement therapy, including

Correspondence to: Professor Lin Sun, Department of Nephrology, The Second Xiangya Hospital, Kidney Institute of Central South University, 139 Renming Road, Changsha, Hunan 410011, P.R. China E-mail: lins2015_0630@163.com

Key words: epithelial-mesenchymal transition, lipopolysaccharide, melatonin, Toll-like receptor 4 hemodialysis (HD), peritoneal dialysis (PD) and kidney transplantation (3). PD and HD are dialysis options for patients with ESRD for whom preemptive kidney transplantation is not an option. There is limited evidence that one form of dialysis is more successful than the other, although patients on PD have significantly improved quality of life in physical and psychological aspects, and have significantly lower mortality rates, compared with patients on HD (4). At present, an increasing number of patients are opting for PD. According to statistics, the number of patients treated with PD increased worldwide between 1997 and 2008, with a 2.5-fold increase in the prevalence of patients on PD in developing countries (5). However, peritoneal fibrosis remains a serious complication of long-term $\mathrm{PD}$, which leads to the failure of peritoneal function and ending of dialysis. A variety of injury-inducing factors contribute to the occurrence of peritoneal fibrosis, including bioincompatible dialysate components, uremic toxins, refractory or recurrent infectious peritonitis and chronic inflammation (6). Chronic inflammation is a major cause of peritoneal fibrosis and dysfunction.

Epithelial-mesenchymal transition (EMT), which has previously been described in chronic inflammatory and fibrogenic diseases, is a conserved process in which polarized, immobile epithelial cells lose tight junctions and associated adherence, and become migratory mesenchymal cells (7). Emerging evidence shows that the EMT of peritoneal mesothelial cells induced by chronic inflammation may be an important process in peritoneal fibrosis (8). LPS is a critical factor, which can induce EMT and the production of extracellular matrix in several tissues and organs (9), therefore, reducing the frequency of peritonitis in patients remains a challenge.

Further investigations are required to identify mechanisms, which can delay or minimize the occurrence of LPS-induced EMT during PD. As an antioxidant, melatonin has antifibrotic properties, although there are limited data providing support for this. Thus, melatonin may be a novel option for inhibiting LPS-induced EMT and assist in reducing the risk of peritoneal fibrosis. The present study aimed to find novel treatment procedures for renal fibrosis using melatonin.

\section{Materials and methods}

Reagents. Ultra-pure LPSs from Escherichia coli (O111:B4) were obtained from Invivogen; Thermo Fisher Scientific, 
Inc. (Waltham, MA, USA). Melatonin, RNase-free DNaseI, DMSO and Triton X-100 were purchased from Sigma-Aldrich; Thermo Fisher Scientific, Inc.). SP600125 and BAY 11-7082 were purchased from Beyotime Institute of Biotechnology (Shanghai, China). Dulbecco's modified Eagle's medium/F12 (DMEM/F12) and fetal bovine serum (FBS) were purchased from Gibco; Thermo Fisher Scientific, Inc. Anti-GAPDH, anti-total (t)-c-Jun N-terminal kinase (JNK), anti-phosphorylated (p)-JNK, anti-vimentin, anti-E-cadherin, anti- $\alpha$-smooth muscle actin (SMA) and anti-Snail antibodies were purchased from Santa Cruz Biotechnology, Inc. (Santa Cruz, CA, USA). The ECL kit was purchased Pierce; Thermo Fisher Scientific, Inc.). All water used was glass distilled.

Cell culture. The human peritoneal mesothelial cell line (HMrSV5) was purchased from the Cell Culture Centre, Chinese Academy of Medical Sciences (Beijing, China). The HMrSV5 cells were cultured in DMEM/F12 medium containing $10 \%$ FBS in a humidified atmosphere consisting of $95 \% \mathrm{O}_{2}$ and $5 \% \mathrm{CO}_{2}$ at $37^{\circ} \mathrm{C}$. The cells were subcultured every 3 days using $0.2 \%$ trypsin and $0.02 \%$ EDTA. For experiments, the cells were seeded at a density of $3 \times 10^{4}$ cells $/ \mathrm{cm}^{2}$ and cultured for $24 \mathrm{~h}$ to obtain monolayers in $3 \mathrm{ml} \mathrm{DMEM} / \mathrm{F} 12$ with $10 \%$ FBS. At $80 \%$ confluence, the cells were exposed to the following conditions: i) control groups, the cells were treated with fresh serum-free DMEM/F12 only; ii) experimental groups, the cells were subjected to pretreatment with $10 \mu \mathrm{g} / \mathrm{ml}$ LPS for 24,48 and $72 \mathrm{~h}$ in the absence or presence of $1 \mu \mathrm{M}$ melatonin at $37^{\circ} \mathrm{C}$ in a humidified atmosphere of $5 \%$ $\mathrm{CO}_{2}$. To further elucidate into this signaling pathway leading to the protection of EMT by melatonin in HMrSV5 cells, $10 \mu \mathrm{mol} / 1 \mathrm{SP} 600125$ or BAY $1177082(10 \mathrm{mM})$ were used. Cells were incubated with or without $10 \mu \mathrm{M}$ of SP600125 or $10 \mathrm{mM}$ BAY 1177082 for $1 \mathrm{~h}$, and then exposed to $10 \mu \mathrm{g} / \mathrm{ml}$ LPS for $48 \mathrm{~h}$ with or without $1 \mu \mathrm{M}$ melatonin pretreatment. For experiments, the cells were cultured for $24 \mathrm{~h}$ to obtain monolayers in $3 \mathrm{ml} \mathrm{DMEM} / \mathrm{F} 12$ with $10 \%$ FBS. Following rinsing of the cells with phosphate-buffered saline (PBS), the medium was replaced and the cells were cultured further.

Immunofluorescence staining. The HMrSV5 cells were seeded into six-well plates for $24 \mathrm{~h}$. Following fixing with ice-cold methanol for $5 \mathrm{~min}$, the cells were permeabilized with $0.1 \%$ Triton-X100 for 5 min, blocked with $1 \%$ bovine serum albumin (Sangon Biotech Co., Ltd., Shanghai, China) for $10 \mathrm{~min}$, and incubated with mouse monoclonal anti-E-cadherin antibody (cat. no. sc-21791; 1:400) or anti-vimentin antibody (cat. no. sc-373717; 1:400) for $2 \mathrm{~h}$ at $37^{\circ} \mathrm{C}$. Following three washes in PBS, the cells were incubated with tetramethylrhodamine isothiocyanate- and fluorescein isothiocyanate-conjugated goat anti-mouse $\operatorname{IgG}$ (1:100 in PBS) for $0.5 \mathrm{~h}$ at room temperature. Subsequently, Hoechst 33342 was added to the cells for $15 \mathrm{~min}$. Following washing three times with PBS, all the samples were examined using optical microscopy (Olympus Corporation, Tokyo, Japan).

Reverse transcription-polymerase chain reaction (RT-PCR) analysis. Total RNA was extracted from the HMrSV5 cells using TRIzol (Tiangen Biotech Co., Ltd., Beijing, China). The RT-PCR kit was purchased from (Takara Bio, Inc., Otsu, Japan).
First-strand cDNA was synthesized from a $1 \mu \mathrm{g}$ aliquot of the total RNA samples using oligo-dT primers and reverse transcriptase. The following primers were used: GAPDH, forward 5-CGGAGTCAACGGATTTGGTCGTAT-3 and reverse 5-AGCCTTCTCCATGGTGGTGAAGAC-3; E-cadherin, forward 5-TTGCTCACATTTCCCAАCTCCTC-3 and reverse 5-CACCTTCAGCCATCCTGTTTCTC-3; Vimentin, forward 5-GCTGAATGACCGCTTCGCCAACT-3 and reverse 5-AGC TCCCGCATCTCCTCCTCGTA-3; $\alpha$-SMA, forward 5-AAG AGGAAGACAGCACAGCTC-3 and reverse 5-TTACAG AGCCCAGAGCCATT-3; Toll-like receptor (TLR)-4, forward 5-TGTCTGAACTCCCTCCAGGT-3 and reverse 5-CACACT GAGGACCGACACAC-3; nuclear factor (NF) $-\kappa B$, forward 5-TGGTGAAGACCTTGCTGCTAAATGC-3 and reverse 5-ACTGGGTGAGGTTGTCTGTCGGTA-3. RT-PCR was performed for 30 cycles. The reactions were performed with a Gene Amp PCR system 9700 (PerkinElmer, Inc., Waltham, MA, USA). The amplified products were separated by electrophoresis on a $2 \%$ agarose gel and visualized by ethidium bromide staining. Each product was visualized following separation and using GAPDH as an internal control. The image density was quantified using a FluoroImager SI (Amersham Pharmacia Biotech, Amersham, UK).

Western blot analysis. The HMrSV5 cells were washed with cold PBS three times and lysed in RIPA buffer (Beyotime Institute of Biotechnology). The cell lysates were harvested by centrifugation at $12,000 \mathrm{~g}$ for $10 \mathrm{~min}$ at $4^{\circ} \mathrm{C}$, and the protein concentration was determined using a Bradford assay (Bio-Rad Laboratories, Inc., Hercules, CA, USA). The samples, containing $10 \mu \mathrm{g}$ of proteins, were electrophoresed on a $10 \%$ SDS-PAGE gel and then transferred onto a polyvinylidene fluoride membrane (EMD Millipore, Billerica, MA, USA). The membranes were incubated at $4^{\circ} \mathrm{C}$ overnight with the following primary antibodies: Anti- $\alpha$-SMA (cat. no. sc-324317; 1:400), anti-E-cadherin (1:400), anti-vimentin (1:400), anti-t-JNK (cat. no. sc-137018; 1:400), anti-p-JNK (cat. no. sc-6254; 1:400), anti-Snail (cat. no. sc-10432; 1:400) and anti-GAPDH (cat. no. sc-365062; 1:1,000). Subsequently, the membranes were incubated with the appropriate peroxidase-conjugated secondary antibodies (cat. no. sc-3795) for $2 \mathrm{~h}$ at room temperature, and detection was performed using an enhanced chemiluminescence kit (Pierce; Thermo Fisher Scientific, Inc.). The relative expression levels of the proteins were analyzed, and the results were quantified using QuantityOne software (Bio-Rad Laboratories, Inc.

Statistical analysis. The data were analyzed statistically using SPSS software 18.0 (IBM SPSS, Armonk, NY, USA) and presented as the mean \pm standard deviation. Analysis was performed using one-way analysis of variance followed by Dunnett's post-hoc test. $\mathrm{P}<0.05$ was considered indicative of a statistically significant difference.

\section{Results}

LPS induces EMT in HMrSV5 cells. LPS is released during the lysis of bacteria. It has been reported that LPS can induce cytokines from immune cells and induce cell apoptosis (10), however, whether LPS induces EMT in HMrSV5 cells remains 


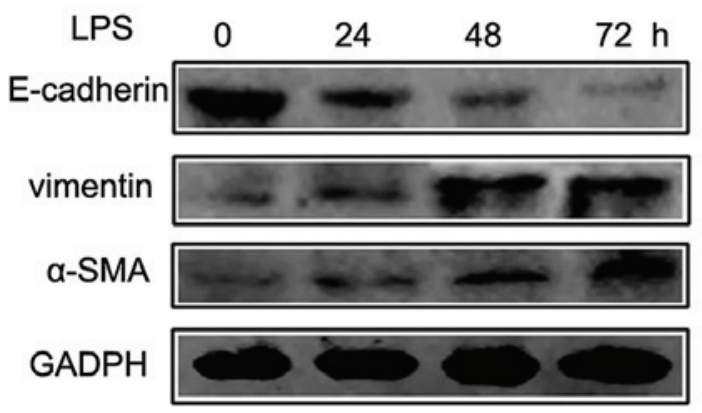

B

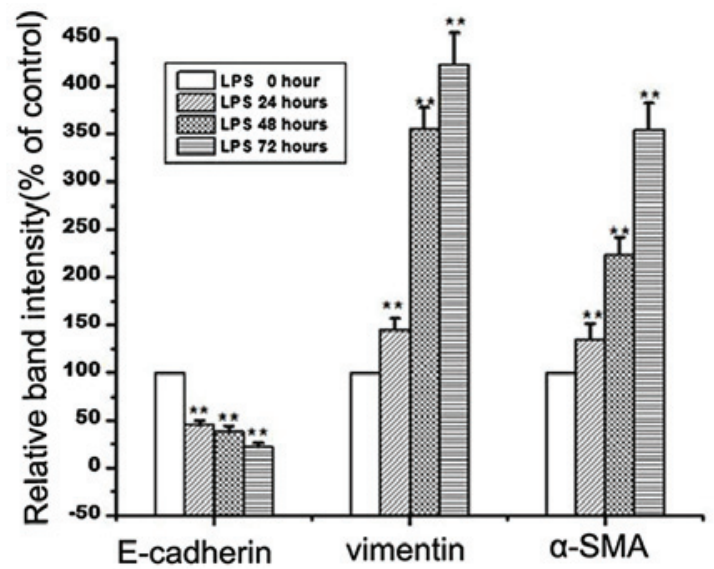

C
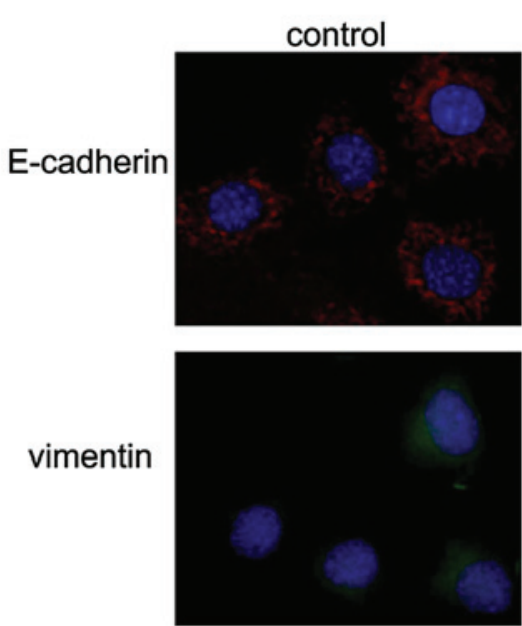
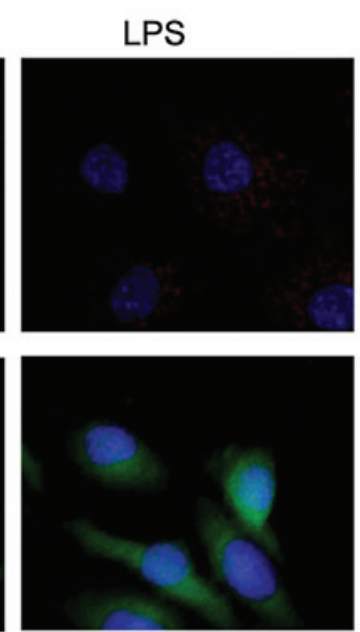

Figure 1. Effect of LPS on the epithelial-mesencyhmal transition of HMrSV5 cells. (A) HMrSV5 cells were cultured with $10 \mu \mathrm{g} / \mathrm{ml}$ LPS for 24,48 and $72 \mathrm{~h}$, and the expression levels of vimentin, E-cadherin and $\alpha$-SMA were detected using western blot analysis. (B) All data are presented as the mean \pm standard deviation ( $\mathrm{n}=6)$. GAPDH protein was used as an internal control ( $\left(^{* *} \mathrm{P}<0.01\right.$, vs. LPS $0 \mathrm{~h}$ group). (C) HMrSV5 cells were cultured with $10 \mu \mathrm{g} / \mathrm{ml} \mathrm{LPS}$ for $24 \mathrm{~h}$, and the expression levels of E-cadherin and vimentin were detected using immunofluorescence. Magnification, x100. LPS, lipopolysaccharide; $\alpha$-SMA, $\alpha$-smooth muscle actin.

to be elucidated. To investigate LPS-induced EMT, in the present study, HMrSV5 cells were treated with $10 \mu \mathrm{g} / \mathrm{ml}$ LPS for 24, 48 and $72 \mathrm{~h}$, and the expression levels of E-cadherin, vimentin and $\alpha$-SMA were detected using western blot analysis and immunofluorescence. The concentration of LPS was selected based on a previous study (11). As shown in Fig. 1A and $\mathrm{B}$, the results of the western blot analysis showed that LPS treatment downregulated the level of E-cadherin, and upregulated the expression levels of vimentin and $\alpha$-SMA. Similarly, immunofluorescence confirmed the that the protein expression of vimentin was increased, and that of E-cadherin was decreased following culture with LPS (Fig. 1C). Collectively, these observations suggested that the HMrSV5 cells had undergone EMT following treatment with LPS.

Melatonin suppresses LPS-induced EMT. Previous reports have shown that melatonin has an anti-inflammatory effect (12). The present study investigated the effects of melatonin on LPS-induced EMT in HMrSV5 cells. The HMrSV5 cells were subjected to $10 \mu \mathrm{g} / \mathrm{ml}$ LPS for $48 \mathrm{~h}$ in the absence or presence of $1 \mu \mathrm{M}$ melatonin. The concentration of melatonin was selected based on a previous study (13), and had no effect on proliferation or apoptosis. The effects of melatonin on LPS-induced EMT of HMrSV5 cells were assessed using western blot and RT-PCR analyses. The EMT induced by LPS was attenuated by co-treating the cells with $1 \mu \mathrm{M}$ melatonin, which was evidenced by the reduced upregulation of $\alpha$-SMA and vimentin, and amelioration of the inhibited expression of E-cadherin at the mRNA (Fig. 2A) and protein (Fig. 2B) levels. These results showed that melatonin supplementation reversed LPS-induced EMT in the HMrSV5 cells.

Melatonin inhibits the LPS-induced activation of TLR4/JNK signaling. TLRs recognize a variety of microbial structural components, termed pathogen-associated molecular patterns. TLR4/JNK signaling is involved in tumor invasion and EMT (14). The present study examined whether melatonin mediated its effects on EMT in HMrSV5 cells through this pathway. The HMrSV5 cells were cultured with $10 \mu \mathrm{g} / \mathrm{ml}$ LPS, with or without $1 \mu \mathrm{M}$ melatonin for $48 \mathrm{~h}$, and the levels of TLR-4, t-JNK and p-JNK were detected using RT-PCR and western blot analyses. The results revealed that LPS upregulated the level of TLR-4 and the phosphorylation of JNK. However, no significant change was observed in the expression of t-JNK (P>0.05; Fig. 3A and B). These results led to the hypothesis that melatonin may suppress EMT in HMrSV5 cells through 
A

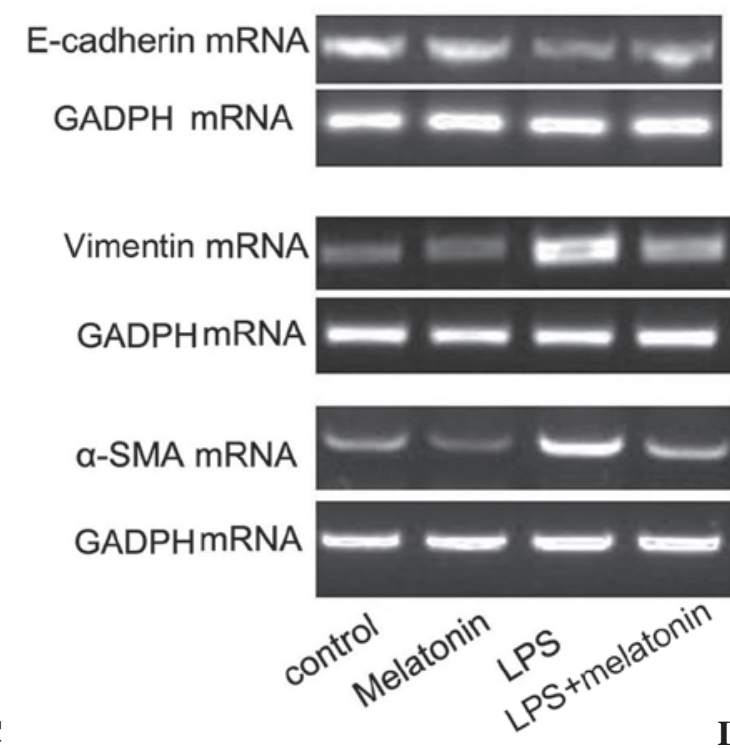

D

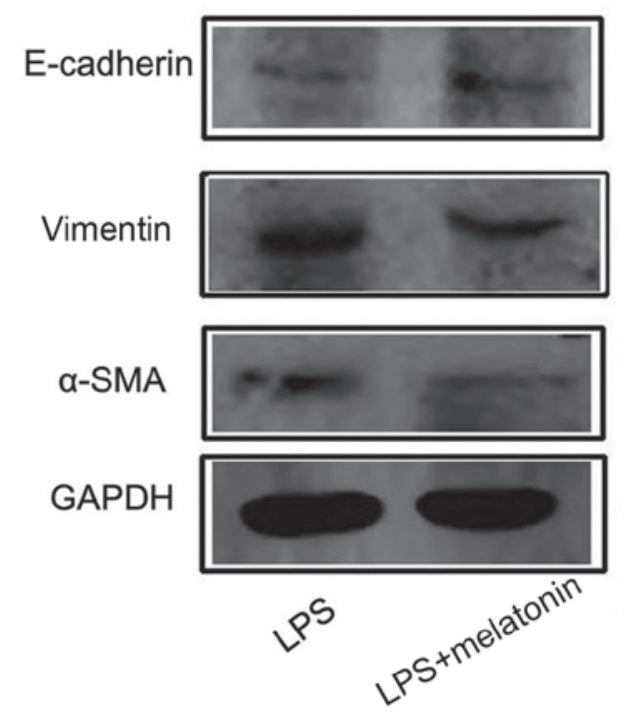

D

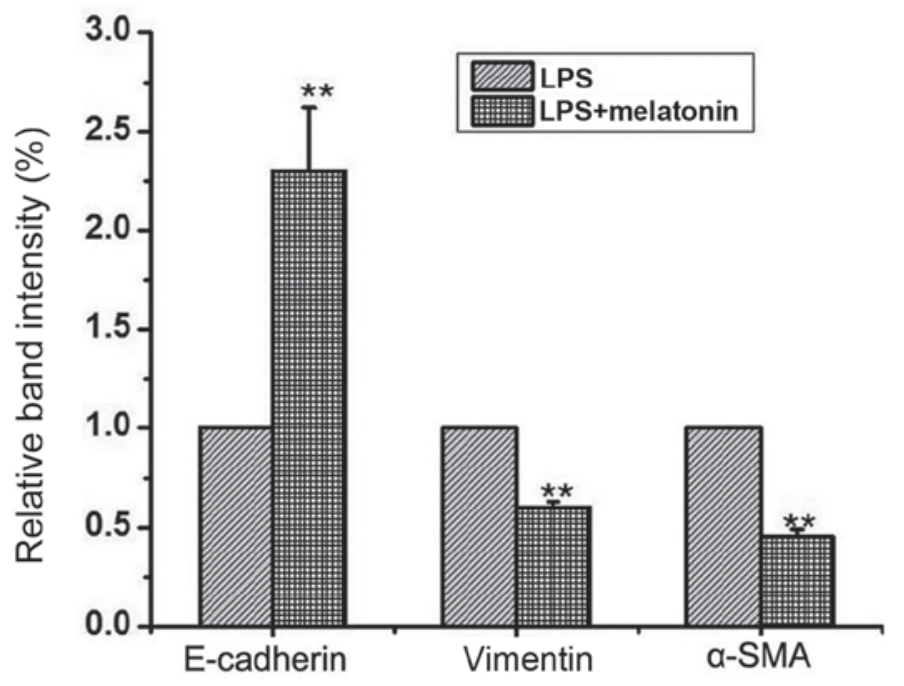

Figure 2. Melatonin inhibits LPS-induced epithelial-mesenchymal transition in HMrSV5 cells. (A) HMrSV5 cells were cultured in $10 \mu$ g/ml LPS with or without $1 \mu \mathrm{M}$ melatonin for $48 \mathrm{~h}$, and the mRNA expression levels of vimentin, E-cadherin and $\alpha$-SMA were detected using reverse transcription-polymerase chain reaction analysis. (B) All data are presented as the mean \pm standard deviation $(n=6)$. GAPDH was used as an internal control $\left({ }^{* *} \mathrm{P}<0.01\right.$, vs. control; ${ }^{\#} \mathrm{P}<0.01 \mathrm{LPS}$, vs. LPS+melatonin). (C) Cells were cultured in $10 \mu \mathrm{g} / \mathrm{ml}$ LPS with or without $1 \mu \mathrm{M}$ melatonin for $48 \mathrm{~h}$, and the expression levels of vimentin, E-cadherin and $\alpha$-SMA were detected using western blot analysis. (D) All data are presented as the mean \pm standard deviation ( $\mathrm{n}=6$ ). GAPDH protein was used as an internal control ( ${ }^{* *} \mathrm{P}<0.01$, vs. LPS). LPS, lipopolysaccharide; $\alpha$-SMA, $\alpha$-smooth muscle actin.

inhibiting the TLR4/JNK pathway. The present study further investigated the effect of melatonin on the TLR4/JNK pathway with $10 \mu \mathrm{mol} / 1 \mathrm{SP} 600125$, an inhibitor of JNK. The concentration of SP600125 was selected based on a previous study (15). From the results of Fig. 3C, The inhibitor of JNK effectively eliminated the protective effect of melatonin, causing a decrease in the level of E-cadherin, and increases in the levels of vimentin and $\alpha$-SMA. Taken together, these findings suggested that the inactivation of the TLR4/JNK pathway was a critical event in the melatonin-induced anti-EMT effect in HMrSV5 cells.

Melatonin inhibits the LPS-induced activation of TLR4/NF- $K B$-Snail signaling pathway. Several studies have reported that TLR4/NF- $\mathrm{B}$ inactivation can protect EMT (16). To further elucidate into this signaling pathway leading to the protection of EMT by melatonin in HMrSV5 cells, a specific

$\mathbf{B}$

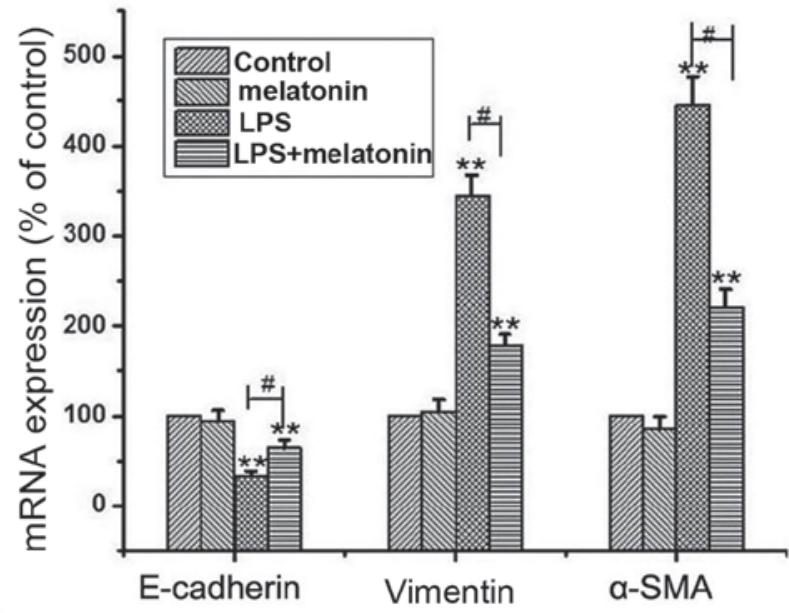


A
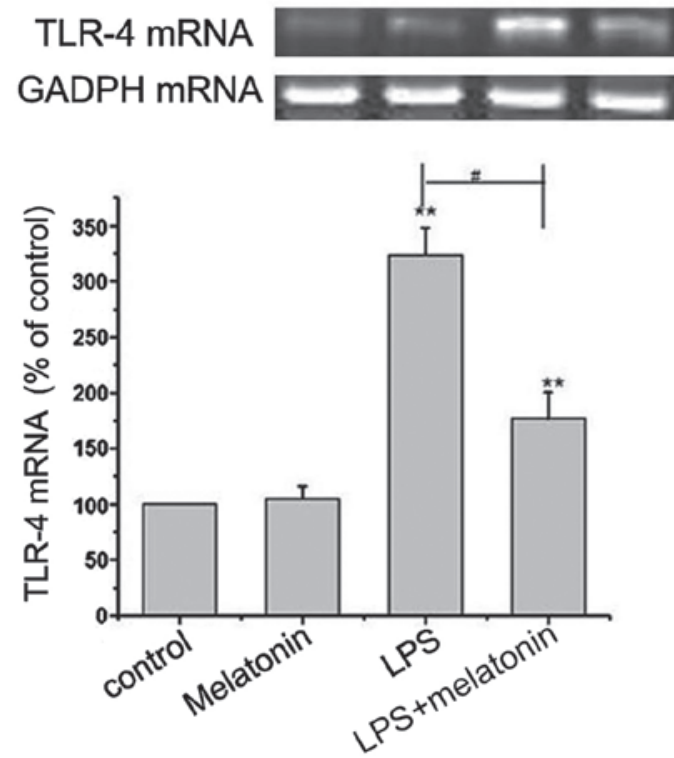

C
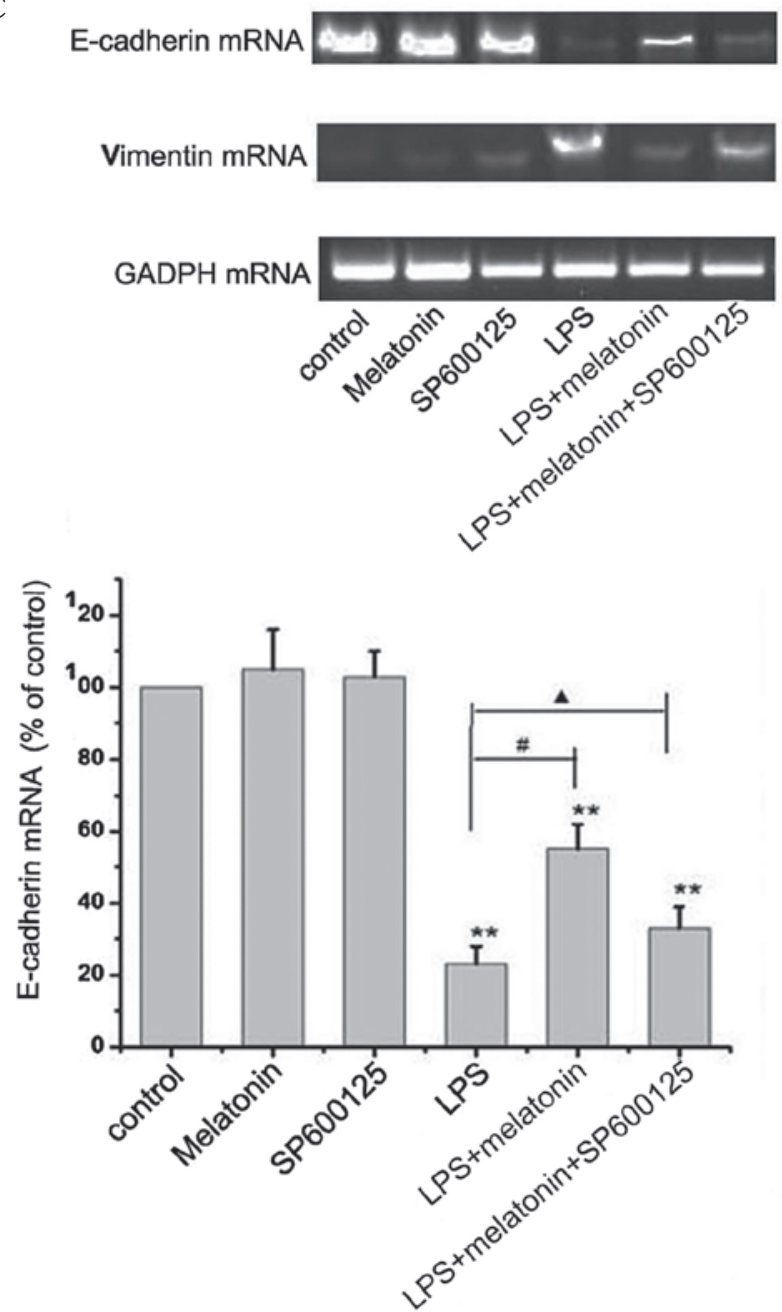

B
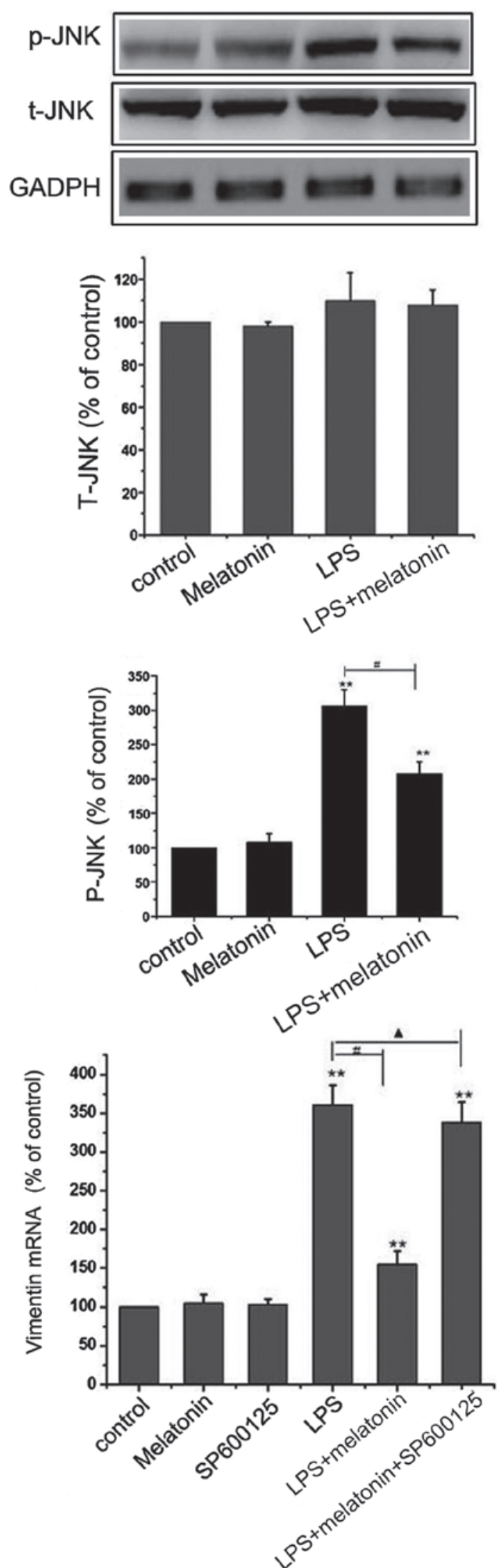

Figure 3. Melatonin inhibits LPS-induced epithelial-mesenchymal transition through the TLR4/JNK signaling pathway. (A) HMrSV5 cells were cultured with $10 \mu \mathrm{g} / \mathrm{ml} \mathrm{LPS}$, with or without $1 \mu \mathrm{M}$ melatonin, for $48 \mathrm{~h}$, and the mRNA level of TLR-4 was detected using RT-PCR analysis. GAPDH was used as an internal control $\left({ }^{* *} \mathrm{P}<0.01\right.$, vs. control; " $\mathrm{P}<0.01 \mathrm{LPS}$, vs. LPS+melatonin). (B) Expression levels of t-JNK and p-JNK were detected using western blot analysis. GAPDH was used as an internal control ( ${ }^{* *} \mathrm{P}<0.01$, vs. control; ${ }^{*} \mathrm{P}<0.01 \mathrm{LPS}$, vs. LPS+melatonin). (C) Cells were incubated with or without $10 \mu \mathrm{M}$ of SP600125 for $1 \mathrm{~h}$, and then exposed to $10 \mu \mathrm{g} / \mathrm{ml}$ LPS for $48 \mathrm{~h}$ with or without $1 \mu \mathrm{M}$ melatonin pretreatment. The mRNA expression levels of vimentin, E-cadherin and $\alpha$-SMA were detected using RT-PCR analyses. Data in all graphs are presented as the mean \pm standard deviation ( $\mathrm{n}=6$ ). GAPDH was used as an internal control $\left({ }^{* *} \mathrm{P}<0.01\right.$, vs. control; ${ }^{*} \mathrm{P}<0.01$ LPS, vs. LPS+melatonin; ${ }^{\mathbf{}} \mathrm{P}>0.05$ LPS+melatonin+SP600125, vs. LPS). LPS, lipopolysaccharide; TLR4, Toll-like receptor 4; JNK, c-Jun N-terminal kinase; T-, total; P-, phosphorylated; $\alpha$-SMA, $\alpha$-smooth muscle actin; RT-PCR, reverse transcription-polymerase chain reaction. 
A
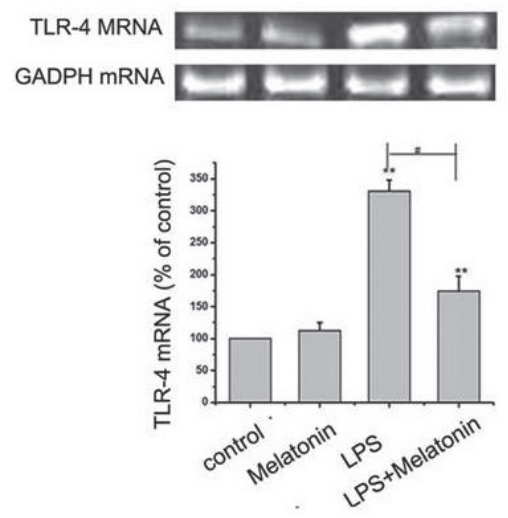

C

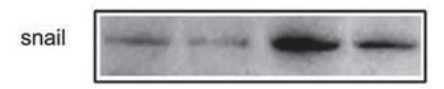

GADPH
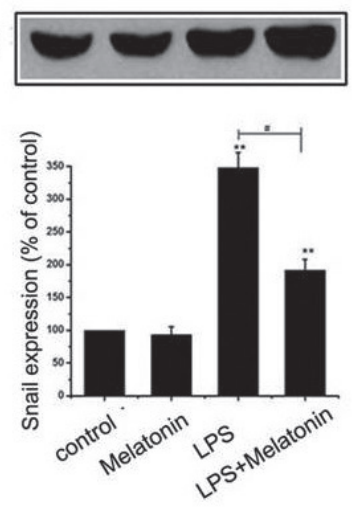

B
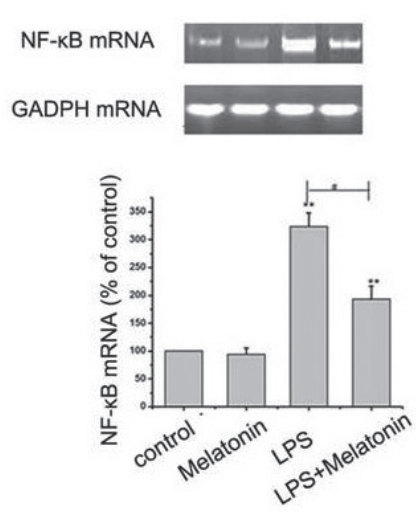

D
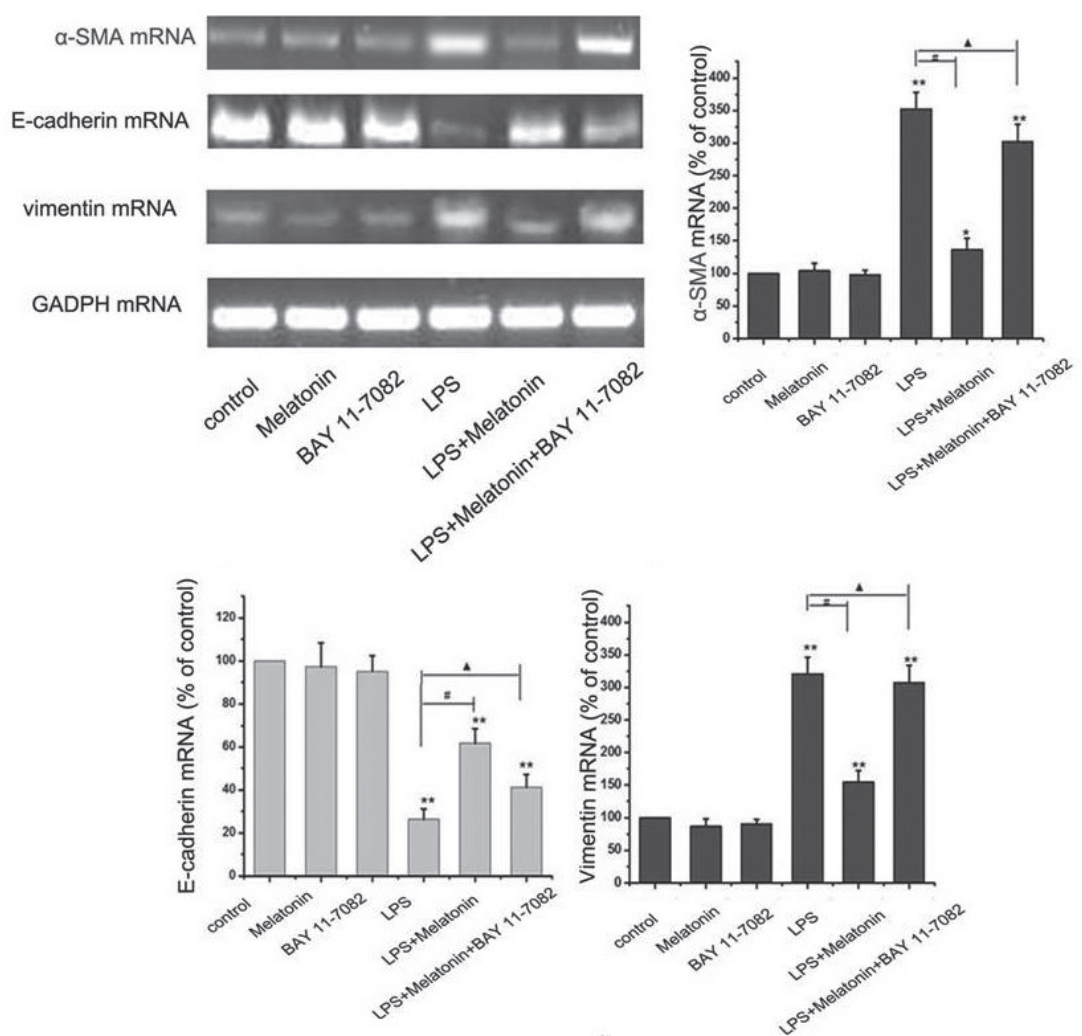

Figure 4. Melatonin inhibits LPS-induced epithelial-mesenchymal transition through the TLR4/NF- $\mathrm{B}$-Snail signaling pathway. (A) HMrSV5 cells were cultured in $10 \mu \mathrm{g} / \mathrm{ml}$ LPS with or without $1 \mu \mathrm{M}$ melatonin for $48 \mathrm{~h}$, and the mRNA expression of TLR4 was detected using RT-PCR analysis. GAPDH was used as an internal control $\left({ }^{* *} \mathrm{P}<0.01\right.$, vs. control; ${ }^{*} \mathrm{P}<0.01 \mathrm{LPS}$, vs. LPS+melatonin). (B) Cells were treated, as described above and the expression of $\mathrm{NF}-\kappa \mathrm{B}$ was detected using RT-PCR analysis. GAPDH was used as an internal control ( ${ }^{* *} \mathrm{P}<0.01$. vs. control; ${ }^{*} \mathrm{P}<0.01 \mathrm{LPS}$ vs, LPS+melatonin). (C) Cells were treated, as described above, and the expression of Snail was detected using western blot analysis. GAPDH was used as an internal control $(* * 2 \times 0.01$, vs. control; ${ }^{\#} \mathrm{P}<0.01 \mathrm{LPS}$, vs. LPS+melatonin). (D) Cells were incubated with or without $10 \mu \mathrm{M}$ of BAY $11-7082$ for $1 \mathrm{~h}$, and then exposed to $10 \mu \mathrm{g} / \mathrm{ml} \mathrm{LPS}$ for $48 \mathrm{~h}$ in the presence or absence of $1 \mu \mathrm{M}$ melatonin pretreatment. The mRNA expression levels of vimentin, E-cadherin and $\alpha$-SMA were detected using RT-PCR analysis. $\left({ }^{* *} \mathrm{P}<0.01\right.$, vs. control; ${ }^{\#} \mathrm{P}<0.01 \mathrm{LPS}$, vs. LPS+melatonin; ${ }^{\mathbf{\Delta}} \mathrm{P}>0.05 \mathrm{LPS}+$ melatonin+BAY $11-7082$, vs. LPS $)$. All data are presented as the mean \pm standard deviation $(n=6)$. GAPDH was used as an internal control EMT, epithelial-mesenchymal transition; LPS, lipopolysaccharide; TLR4, Toll-like receptor 4; $\alpha$-SMA, $\alpha$-smooth muscle actin; NF- $\kappa \mathrm{B}$, nuclear factor- $\kappa \mathrm{B}$; RT-PCR, reverse transcription-polymerase chain reaction.

nucleus (18). Melatonin regulates several physiological functions, including sleep and circadian rhythms, immune function, body weight and energy balance, and has anticancer effects $(19,20)$. In addition, as a potent antioxidant, melatonin has been found to be effective in a variety of disorders linked to oxidative stress and inflammation (21). Several studies have demonstrated that melatonin alleviates the LPS-induced expression of inflammatory cytokines/chemokines. Melatonin has been found to regulate LPS-induced proinflammatory and anti-inflammatory cytokines in the serum, fluid, liver and brain (22). Melatonin inhibits the production of NO and protects neural stem cells against LPS-induced inflammatory stress (23). Melatonin also reduces the expression of pro-inflammatory mediators and enhances the expression of heme oxygenase 1 via the NF- $\kappa B, p 38$ mitogen-activated protein kinase and nuclear factor erythroid 2 related factor 2 cascade signaling pathways in murine macrophages (24). In the present study, it was confirmed that melatonin was associated with anti-inflammation effects and has a protective role in LPS-induced EMT in HMrSV5 cells, reducing the 
upregulation of $\alpha$-SMA and vimentin, and ameliorating the expression of E-cadherin (Fig. 2).

LPS-induced inflammation and LPS-induced EMT are key in peritoneal fibrosis in PD. A previous study found that exposure to LPS in a rat model led to peritoneal fibrosis and neoangiogenesis (25). TLR4 is responsible for the immediate response to Gram-negative bacteria and is recruited to initiate a signaling cascade leading to production of pro-inflammatory cytokines. The presents study investigated the expression of TLR4 during LPS stimulation. A shown in Fig. 3, LPS significantly upregulated the level of TLR4, determined using RT-PCR. A previous study confirmed this result, in which LPS/TLR4 signaling led to the activation of the transcription factors, NF- $\kappa \mathrm{B}$, IL- 6 and TNF- $\alpha$ (26). In the present study, it was confirmed that melatonin was associated with TLR4/JNK and TLR4/NF- $\kappa$ B-Snail inactivation in LPS-treated HMrSV5 cells (Figs. 3 and 4). Therefore, it is possible that melatonin suppresses LPS-induced EMT via inhibiting the TLR4/JNK and TLR4/NF- $\kappa$ B-Snail pathways. Therefore, targeting inflammation, EMT or associated cell signaling pathways may inhibit peritoneal fibrosis in PD. The findings of the present study indicated that melatonin offers potential in the prevention and therapy of peritoneal fibrosis.

\section{References}

1. Rastogi A, Linden A and Nissenson AR: Disease management in chronic kidney disease. Adv Chronic Kidney Dis 15: 19-28, 2008.

2. Zelmer JL: The economic burden of end-stage renal disease in Canada. Kidney Int 72: 1122-1129, 2007.

3. Knoll GA and Nichol G: Dialysis, kidney transplantation, or pancreas transplantation for patients with diabetes mellitus and renal failure: A decision analysis of treatment options. J Am Soc Nephrol 14: 500-515, 2003.

4. Beladi Mousavi SS, Hayati F, Valavi E, Rekabi F and Mousavi MB: Comparison of survival in patients with end-stage renal disease receiving hemodialysis versus peritoneal dialysis Saudi J Kidney Dis Transpl 26: 392-397, 2015.

5. Jain AK, Blake P, Cordy P and Garg AX: Global trends in rates of peritoneal dialysis. J Am Soc Nephrol 23: 533-544, 2012.

6. Ueno T, Nakashima A, Doi S, Kawamoto T, Honda K, Yokoyama Y, Doi T, Higashi Y, Yorioka N, Kato Y, et al: Mesenchymal stem cells ameliorate experimental peritoneal fibrosis by suppressing inflammation and inhibiting TGF- $\beta 1$ signaling. Kidney Int 84: 297-307, 2013.

7. Puisieux A, Brabletz T and Caramel J: Oncogenic roles of EMT-inducing transcription factors. Nat Cell Biol 16: 488-494, 2014.

8. Strippoli R, Moreno-Vicente R, Battistelli C, Battistelli C, Cicchini C, Noce V, Amicone L, Marchetti A, Del Pozo MA and Tripodi M: Molecular mechanisms underlying peritoneal EMT and fibrosis. Stem Cells Int: 3543678, 2016.

9. Chen MC, Chang WW, Kuan YD, Lin ST, Hsu HC and Lee CH: Resveratrol inhibits LPS-induced epithelial-mesenchymal transition in mouse melanoma model. Innate Immun 18: 685-693, 2012.
10. Huang C, Zheng H, He W, Lu G, Li X, Deng Y, Zeng M Ghrelin ameliorates the human alveolar epithelial A549 cell apoptosis induced by lipopolysaccharide. Biochem Biophys Res Commun 474: 83-90, 2016

11. Jing YY, Han ZP, Sun K, Zhang SS, Hou J, Liu Y, Li R, Gao L, Zhao X, Zhao QD, et al: Toll-like receptor 4 signaling promotes epithelial-mesenchymal transition in human hepatocellular carcinoma induced by lipopolysaccharide. BMC Med 10: 98 , 2012.

12. Esposito E and Cuzzocrea S: Antiinflammatory activity of melatonin in central nervous system. Curr Neuropharmacol 8: 228-242, 2010

13. Obana-Koshino A, Ono H, Miura J, Sakai M, Uchida H, Nakamura W, Nohara K, Maruyama Y, Hattori A and Sakai T: Melatonin inhibits embryonic salivary gland branching morphogenesis by regulating both epithelial cell adhesion and morphology. PLoS One 10: e0119960, 2015.

14. Pirianov G, Torsney E, Howe F and Cockerill GW: Rosiglitazone negatively regulates c-Jun $\mathrm{N}$-terminal kinase and toll-like receptor 4 proinflammatory signalling during initiation of experimental aortic aneurysms. Atherosclerosis 225: 69-75, 2012

15. Xiong XX, Liu JM, Qiu XY, Pan F, Yu SB and Chen XQ: Piperlongumine induces apoptotic and autophagic death of the primary myeloid leukemia cells from patients via activation of ROS-p38/JNK pathways. Acta Pharmacol Sin 36: 362-374, 2015.

16. Naugler WE and Karin M: NF-kappaB and cancer-identifying targets and mechanisms. Curr Opin Genet Dev 18: 19-26, 2008.

17. Gu K, Li MM, Shen J, Liu F, Cao JY, Jin S and Yu Y: Interleukin-17-induced EMT promotes lung cancer cell migration and invasion via NF- $\mathrm{KB} / \mathrm{ZEB} 1$ signal pathway. Am J Cancer Res 5: 1169-1179, 2015.

18. Houdek P, Polidarová L, Nováková M, Matějů K, Kubík Š and Sumová A: Melatonin administered during the fetal stage affects circadian clock in the suprachiasmatic nucleus but not in the liver. Dev Neurobiol 75: 131-144, 2015.

19. De Pedro N, Martínez-Alvarez RM and Delgado MJ: Melatonin reduces body weight in goldfish (Carassius auratus): Effects on metabolic resources and some feeding regulators. J Pineal Res 45: 32-39, 2008.

20. Plaimee P, Weerapreeyakul N, Barusrux S and Johns NP: Melatonin potentiates cisplatin-induced apoptosis and cell cycle arrest in human lung adenocarcinoma cells. Cell Prolif 48: 67-77, 2015.

21. Carrasco C, Marchena AM, Holguín-Arévalo MS, Martín-Partido G, Rodríguez AB, Paredes SD and Pariente JA: Anti-inflammatory effects of melatonin in a rat model of caerulein-induced acute pancreatitis. Cell Biochem Funct 31: 585-590, 2013.

22. Zhao H, Wu QQ, Cao LF, Qing HY, Zhang C, Chen YH, Wang H, Liu RY and Xu DX: Melatonin inhibits endoplasmic reticulum stress and epithelial-mesenchymal transition during bleomycin-induced pulmonary fibrosis in mice. PLoS One 9: e97266, 2014.

23. Song J, Kang SM, Lee KM and Lee JE: The protective effect of melatonin on neural stem cell against LPS-induced inflammation. Biomed Res Int 2015: 854359, 2015.

24. Aparicio-Soto M, Alarcón-de-la-Lastra C, Cárdeno A, Sánchez-Fidalgo $\mathrm{S}$ and Sanchez-Hidalgo M: Melatonin modulates microsomal PGE synthase 1 and NF-E2-related factor-2-regulated antioxidant enzyme expression in LPS-induced murine peritoneal macrophages. Br J Pharmacol 171: 134-144, 2014.

25. Margetts PJ, Kolb M, Yu L, Hoff CM and Gauldie J: A chronic inflammatory infusion model of peritoneal dialysis in rats. Perit Dial Int 21 (Suppl 3): S368-S372, 2001.

26. Nijland R, Hofland T and van Strijp JA: Recognition of LPS by TLR4: Potential for anti-inflammatory therapies. Mar Drugs 12: 4260-4273, 2014. 